\title{
Corollaries and multiple extensions of Gessel and Stanton hypergeometric summation formulas
}

\author{
Thomas Ernst and Per W. Karlsson
}

\begin{abstract}
We find some new simple hypergeometric formulas in the footsteps of the important article by Gessel and Stanton. These are multiple reduction formulas, multiple summation formulas, as well as multiple transformation formulas for special Kampé de Fériet functions and Appell functions. The hypergeometric summation formulas have special function arguments in $\mathbb{Q}$ and parameter values in $\mathbb{N}$ or $\mathbb{C}$. The proofs use Pfaff-Kummer transformation, Euler transformation, or an improved form of Slater reversion.
\end{abstract}

\section{Introduction}

The purpose of this article is to extend the formulas in the article by Gessel and Stanton [2] to more hypergeometric and multiple hypergeometric formulas. It is based on the notes of the late Per Karlsson, whose son kindly provided them to the first author. All the new formulas have been checked by Mathematica. All the computations in the second part of the paper begin with certain parts of [2]. We have skipped the beginning of that article and just refer to the corresponding formulas. We begin with some multiple hypergeometric formulas in Section 2, where the proofs by generating functions are very similar. Then several corollaries of $[2, \S 3,6]$ are listed in Sections 3 and 4. The structure of presentation of the latter formulas always starts with the formula number in [2] and some of the formulas could even be conjectures. The reversion formula, which we will use several times, is a corrected form a formula of Slater [7, p. 48]:

$$
{ }_{2} \mathrm{~F}_{1}\left[\begin{array}{c|c}
a,-m \\
b
\end{array} \mid z\right]=\frac{(a)_{m}}{(b)_{m}}(-z)^{m}{ }_{2} \mathrm{~F}_{1}\left[\begin{array}{c}
1-b-m,-m \\
1-a-m
\end{array} \mid z^{-1}\right] .
$$

Received March 10, 2020.

2020 Mathematics Subject Classification. Primary 33C65; Secondary 33C05, 33B15.

Key words and phrases. Simple and multiple hypergeometric formulas, reversion, Pfaff-

Kummer and Euler transformations.

https://doi.org/10.12697/ACUTM.2021.25.02 
Throughout, unit arguments in hypergeometric functions are left out [1] and $m, n \in \mathbb{N}$. We shall use two Appell functions.

Definition 1 (see [8]). The second and third Appell functions are

$$
\begin{aligned}
\mathrm{F}_{2}\left[\begin{array}{c}
a ; b, b^{\prime} \\
c, c^{\prime}
\end{array} \mid x_{1}, x_{2}\right] \equiv & \sum_{m_{1}, m_{2}=0}^{\infty} \frac{(a)_{m_{1}+m_{2}}(b)_{m_{1}}\left(b^{\prime}\right)_{m_{2}}}{m_{1} ! m_{2} !(c)_{m_{1}}\left(c^{\prime}\right)_{m_{2}}} x_{1}^{m_{1}} x_{2}^{m_{2}}, \\
& \left|x_{1}\right|+\left|x_{2}\right|<1 . \\
\mathrm{F}_{3}\left(a, a^{\prime} ; b, b^{\prime} ; c ; x_{1}, x_{2}\right) \equiv & \sum_{m_{1}, m_{2}=0}^{\infty} \frac{(a)_{m_{1}}\left(a^{\prime}\right)_{m_{2}}(b)_{m_{1}}\left(b^{\prime}\right)_{m_{2}}}{m_{1} ! m_{2} !(c)_{m_{1}+m_{2}}} x_{1}^{m_{1}} x_{2}^{m_{2}}, \\
& \max \left(\left|x_{1}\right|,\left|x_{2}\right|\right)<1 .
\end{aligned}
$$

Definition 2 (see [8]). The special double Kampé de Fériet function is defined by

$$
\mathrm{F}_{2: 0}^{1: 2}\left[\begin{array}{c|c}
a: b_{1}, b_{2} ; c_{1}, c_{2} \\
d_{1}, d_{2}
\end{array} \mid x_{1}, x_{2}\right] \equiv \sum_{m_{1}, m_{2}=0}^{\infty} \frac{(a)_{m_{1}+m_{2}}\left(b_{1}, b_{2}\right)_{m_{1}}\left(c_{1}, c_{2}\right)_{m_{2}}}{m_{1} ! m_{2} !\left(d_{1}, d_{2}\right)_{m_{1}+m_{2}}} x_{1}^{m_{1}} x_{2}^{m_{2}} .
$$

\section{New multiple hypergeometric formulas}

We will now make several similar computations with generating functions. The proofs of the multiple hypergeometric formulas are made by equating coefficients in obvious double sums. One has

$$
\begin{aligned}
f & (x, y):=(1-x)^{p}(1-y)^{q}\left[1-\frac{A x+B y}{(1-x)(1-y)}\right]^{-a} \\
= & (1-x)^{p}(1-y)^{q} \sum_{i, j=0}^{\infty} \frac{(a)_{i+j}}{i ! j !} \frac{A^{i} x^{i} B^{j} y^{j}}{(1-x)^{i+j}(1-y)^{i+j}} \\
= & \sum_{i, j=0}^{\infty} \frac{(a)_{i+j} A^{i} x^{i} B^{j} y^{j}}{i ! j !}(1-x)^{-i-j+p}(1-y)^{-i-j+q} \\
= & \sum_{i, j, k, l=0}^{\infty} \frac{(a)_{i+j} A^{i} B^{j} x^{i+k} y^{j+l}}{i ! j ! k ! l !}(i+j-p)_{k}(i+j-q)_{l} \\
= & \sum_{i, j, k, l=0}^{\infty} \frac{(a)_{i+j} A^{i} B^{j} x^{i+k} y^{j+l}}{i ! j ! k ! l !(-p)_{i+j}(-q)_{i+j}}(-p)_{i+j+k}(-q)_{i+j+l} \\
= & \sum_{m, n=0}^{\infty} \frac{x^{m} y^{n}(-p)_{m}(-q)_{n}}{m ! n !} \\
& \sum_{i, j=0}^{\infty} \frac{(a)_{i+j}(-m)_{i} A^{i}(-n)_{j} B^{j}(-1)^{i+j}(-p+m)_{j}(-q+n)_{i}}{i ! j !(-p)_{i+j}(-q)_{i+j}} .
\end{aligned}
$$


Thus we have

$$
\begin{aligned}
f(x, y)= & \sum_{m, n=0}^{\infty} \frac{x^{m} y^{n}(-p)_{m}(-q)_{n}}{m ! n !} \\
& \mathrm{F}_{2: 0}^{1: 2}\left[\begin{array}{c}
a:-m,-q+n ;-n,-p+m \\
-p ;-q
\end{array} \mid-A,-B\right] .
\end{aligned}
$$

Theorem 2.1. A reduction formula for a Kampé de Fériet function is

$$
\begin{aligned}
\mathrm{F}_{2: 0}^{1: 2} & {\left[\begin{array}{c}
a:-m,-q+n ;-n,-p+m \\
-p ;-q
\end{array}\right.} \\
& { }_{2} \mathrm{~F}_{1}\left[\begin{array}{c}
-m,-a-p \\
1-a-m
\end{array} \mid-1\right]=\frac{(-1)^{m+n}(a)_{m}(a)_{n}}{(-p)_{m}(-q)_{n}} \\
& \min [p, q] \geq m+n .
\end{aligned}
$$

Proof. For $A=B=-2$, similarly to (2) we have

$$
\begin{aligned}
& f(x, y)=(1-x)^{p+a}(1-y)^{q+a}(1+x)^{-a}(1+y)^{-a}=\sum_{m, n=0}^{\infty} \frac{x^{m} y^{n}}{m ! n !} \\
& \sum_{i, j=0}^{\infty} \frac{(-a-p)_{i}(a)_{m-i}(-a-q)_{j}(a)_{n-j}(-1)^{i+j+k+l}(-m)_{i}(-n)_{j}}{i ! j !} \\
&= \sum_{m, n=0}^{\infty} \frac{x^{m} y^{n}}{m ! n !}(-1)^{m}(-1)^{n}(a)_{m}(a)_{n} \\
& \sum_{i=0}^{\infty} \frac{(-m)_{i}(a+m)_{-i}(-a-p)_{i}}{i !} \sum_{j=0}^{\infty} \frac{(-n)_{j}(a+n)_{-j}(-a-q)_{j}}{j !} \\
&= \sum_{m, n=0}^{\infty} \frac{x^{m} y^{n}}{m ! n !}(-1)^{m}(-1)^{n}(a)_{m}(a)_{n} \\
&{ }_{2} \mathrm{~F}_{1}\left[\begin{array}{c}
-m,-a-p \\
1-a-m
\end{array} \mid-1\right]{ }_{2} \mathrm{~F}_{1}\left[\begin{array}{c}
-n,-a-q \\
1-a-n
\end{array} \mid-1\right] .
\end{aligned}
$$

Finally, equate formulas (3) and (5) to get (4).

Corollary 2.2. A summation formula for a Kampé de Fériet function is

$$
\begin{aligned}
& \mathrm{F}_{2: 0}^{1: 2}\left[\begin{array}{c|c}
a:-m, 2 a+n ;-n, 2 a+m & 2,2 \\
2 a, 2 a &
\end{array}\right] \\
& = \begin{cases}0, & m \text { odd or } n \text { odd, } \\
\frac{\left(\frac{1}{2}\right)_{\frac{1}{2} m}\left(\frac{1}{2}\right)_{\frac{1}{2} n}}{\left(a+\frac{1}{2}\right)_{\frac{1}{2} m}\left(a+\frac{1}{2}\right)_{\frac{1}{2} n}}, & m \text { even and } n \text { even. }\end{cases}
\end{aligned}
$$


Proof. Reversing gives

$$
{ }_{2} \mathrm{~F}_{1}\left[\begin{array}{c|c}
-m,-a-p \\
1-a-m
\end{array} \mid-1\right]=\frac{(-a-p)_{m}}{(1-a-m)_{m}}{ }_{2} \mathrm{~F}_{1}\left[\begin{array}{c}
-m, a \\
1+a+p-m
\end{array} \mid-1\right] .
$$

Using (3), it follows that

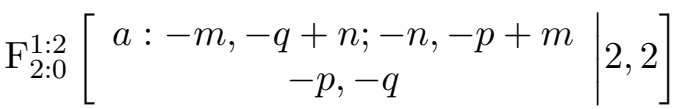

$$
\begin{aligned}
& =\frac{(-a-p)_{m}}{(-p)_{m}} \frac{(-a-q)_{n}}{(-q)_{n}} \\
& { }_{2} \mathrm{~F}_{1}\left[\begin{array}{c|c}
-m, a \\
1+a+p-m
\end{array} \mid-1\right]{ }_{2} \mathrm{~F}_{1}\left[\begin{array}{c}
-n, a \\
1+a+q-n
\end{array} \mid-1\right] .
\end{aligned}
$$

Furthermore, for $p=q=-2 a$ we get

$$
\begin{aligned}
& \mathrm{F}_{2: 0}^{1: 2}\left[\begin{array}{c|c}
a:-m, 2 a+n ;-n, 2 a+m & 2,2 \\
2 a, 2 a &
\end{array}\right] \\
& =\frac{(a)_{m}}{(-p)_{m}} \frac{(a)_{n}}{(-q)_{n}}{ }_{2} \mathrm{~F}_{1}\left[\begin{array}{c}
-m, a \\
1-a-m
\end{array} \mid-1\right]{ }_{2} \mathrm{~F}_{1}\left[\begin{array}{c}
-n, a \\
1-a-n
\end{array} \mid-1\right] .
\end{aligned}
$$

By Kummer's first summation formula and Legendre duplication $\star$ we have

$$
\begin{aligned}
& { }_{2} \mathrm{~F}_{1}\left[\begin{array}{c}
-m, a \\
1-a-m
\end{array} \mid-1\right] \stackrel{\text { by }}{=} 2^{m} \Gamma\left[\begin{array}{c}
1-m-a, \frac{1}{2} \\
1-\frac{1}{2} m-a, \frac{1}{2}-\frac{1}{2} m
\end{array}\right] \\
& = \begin{cases}0, & m \text { odd }, \\
2^{2 k} \frac{(1-a)_{-2 k}}{\left(1-a, \frac{1}{2}\right)_{-k}}=2^{2 k} \frac{\left(a, \frac{1}{2}\right)_{k}}{(a)_{2 k}}=\frac{\left(a, \frac{1}{2}\right)_{k}}{\left(\frac{1}{2} a, \frac{1}{2} a+\frac{1}{2}\right)_{k}}, & m=2 k .\end{cases}
\end{aligned}
$$

By (7), (8) implies (6).

In general, we have

$$
\begin{aligned}
& \mathrm{F}_{2: 0}^{1: 2}\left[\begin{array}{c}
a:-m, d+n ;-n, c+m \\
c, d
\end{array} \mid 2,2\right] \\
& \quad=\frac{(c-a)_{m}(d-a)_{n}}{(c)_{m}(d)_{n}}{ }_{2} \mathrm{~F}_{1}\left[\begin{array}{c}
-m, a \\
1+a-c-m
\end{array} \mid-1\right]{ }_{2} \mathrm{~F}_{1}\left[\begin{array}{c}
-n, a \\
1+a-d-n
\end{array} \mid-1\right] .
\end{aligned}
$$

Putting $d=a$ and reversing, we obtain a "reduction formula" for the third Appell function:

$$
\mathrm{F}_{3}[-m, d+n ;-n, c+m ; c ; 2,2]=\frac{(d)_{m}(-1)^{m+n}}{(c)_{m}}{ }_{2} \mathrm{~F}_{1}\left[\begin{array}{c}
-m,-d+c \\
1-d-m
\end{array} \mid-1\right] .
$$


Theorem 2.3. A transformation formula for the second Appell function is

$$
\begin{aligned}
& \mathrm{F}_{2}\left[\begin{array}{c}
a ;-m,-n \\
c, d
\end{array} \mid z, 1-z\right] \\
& =\frac{(c-a)_{m}(d-a)_{n}}{(c)_{m}(d)_{n}} \mathrm{~F}_{2}\left[\begin{array}{c}
a:-m,-n \\
1+a-c-m, 1+a-d-n
\end{array} \mid 1-z, z\right] .
\end{aligned}
$$

Proof. A similar computation as before gives

$$
\begin{aligned}
g(x, y) & :=(1-x)^{p}(1-y)^{q}\left[1-\frac{A x}{1-x}-\frac{B y}{1-y}\right]^{-a} \\
& =(1-x)^{p}(1-y)^{q} \sum_{i, j=0}^{\infty} \frac{(a)_{i+j}}{i ! j !} \frac{A^{i} x^{i} B^{j} y^{j}}{(1-x)^{i}(1-y)^{j}} \\
& =\sum_{i, j=0}^{\infty} \frac{(a)_{i+j} A^{i} x^{i} B^{j} y^{j}}{i ! j !}(1-x)^{-i+p}(1-y)^{-j+q} \\
& =\sum_{i, j, k, l=0}^{\infty} \frac{(a)_{i+j} A^{i} B^{j} x^{i+k} y^{j+l}}{i ! j ! k ! l !}(i-p)_{k}(j-q)_{l} \\
& =\sum_{i, j, k, l=0}^{\infty} \frac{(a)_{i+j} A^{i} B^{j} x^{i+k} y^{j+l}}{i ! j ! k ! l !(-p)_{i}(-q)_{j}}(-p)_{i+k}(-q)_{j+l} \\
= & \sum_{m, n, i, j=0}^{\infty} \frac{x^{m} y^{n}(-p)_{m}(-q)_{n}}{m ! n !} \frac{(a)_{i+j}(-m)_{i}(-A)^{i}(-n)_{j}(-B)^{j}}{i ! j !(-p)_{i}(-q)_{j}}
\end{aligned}
$$

and so,

$$
g(x, y)=\sum_{m, n=0}^{\infty} \frac{x^{m} y^{n}(-p)_{m}(-q)_{n}}{m ! n !} \mathrm{F}_{2}\left[\begin{array}{c}
a:-m,-n \\
-p,-q
\end{array} \mid-A,-B\right] .
$$

If $A+B=-1$, then

$$
\begin{aligned}
g & (x, y)=(1-x)^{p+a}(1-y)^{q+a}(1-y-x+x y-A x+A x y-B y+B x y)^{-a} \\
= & (1-x)^{p+a}(1-y)^{q+a}(1-(1+A) x-(1+B) y)^{-a} \\
= & (1-x)^{p+a}(1-y)^{q+a}(1+B x+A y)^{-a} \\
= & \sum_{i, j, k, l=0}^{\infty} \frac{(-a-p)_{i}(-a-q)_{j}(a)_{k+l}(-B x)^{k}(-A y)^{l} x^{i} y^{j}}{i ! j ! k ! l !}=\sum_{m, n=0}^{\infty} \frac{x^{m} y^{n}}{m ! n !} \\
& \sum_{k, l=0}^{\infty} \frac{(a)_{k+l}(-B)^{k}(-A)^{l}(-1)^{k+l}(-m)_{k}(-n)_{l}(-a-p)_{m-k}(-a-q)_{n-l}}{k ! l !}
\end{aligned}
$$


which shows that

$$
\begin{aligned}
g(x, y)= & \sum_{m, n=0}^{\infty} \frac{x^{m} y^{n}(-a-p)_{m}(-a-q)_{n}}{m ! n !} \\
& \mathrm{F}_{2}\left[\begin{array}{c}
a:-m,-n \\
1+a+p-m, 1+a+q-n
\end{array} \mid-B,-A\right] .
\end{aligned}
$$

Finally, to get (10), we equate the coefficients in formulas (11) and (12).

Theorem 2.4. A reduction formula for the second Appell function is

$$
\begin{aligned}
& \mathrm{F}_{2}\left[\begin{array}{c|c}
a ;-m,-n \\
c, d
\end{array} \mid 1,1\right] \\
& =\frac{(c-a)_{m}(d-a)_{n}}{(c)_{m}(d)_{n}}{ }_{3} \mathrm{~F}_{2}\left[\begin{array}{c}
a,-m,-n \\
1+a-c-m, 1+a-d-n
\end{array}\right] .
\end{aligned}
$$

Proof. Put $A=B=-1$ in $g(x, y)$ to obtain

$$
\begin{aligned}
g(x, y) & =(1-x)^{p+a}(1-y)^{q+a}(1-x y)^{-a} \\
& =\sum_{i, j, k=0}^{\infty} \frac{(-a-p)_{i}(-a-q)_{j}(a)_{k} x^{k} y^{k} x^{i} y^{j}}{i ! j ! k !} \\
& =\sum_{m, n=0}^{\infty} x^{m} y^{n} \sum_{k=0}^{\infty} \frac{(a)_{k}(-a-p)_{m-k}(-a-q)_{n-k}}{(m-k) ! k !(n-k) !} \\
& =\sum_{m, n=0}^{\infty} \frac{x^{m} y^{n}(-a-p)_{m}(-a-q)_{n}}{m ! n !} \\
& \sum_{k=0}^{\infty} \frac{(a,-m,-n)_{k}}{(1+a+p-m, 1+a+q-n)_{k} k !} \\
= & \sum_{m, n=0}^{\infty} \frac{x^{m} y^{n}(-a-p)_{m}(-a-q)_{n}}{m ! n !} \\
& { }_{3} \mathrm{~F}_{2}\left[\begin{array}{c}
1+a+p-m,-n \\
1+a+q
\end{array}\right] .
\end{aligned}
$$

Finally equate the coefficients in formulas (11) and (14).

Remark 1. Formula (13) has an equivalent version in [5] and in [9]. It also appears for $c=1+d-a$, i.e., balanced in [4].

For finite sums, $\mathrm{F}_{p}(0 ; 4,5)$ can be transformed to $\mathrm{F}_{p}(5 ; 0,1)$, and we obtain the following result. 
Corollary 2.5. A transformation formula for a ${ }_{3} \mathrm{~F}_{2}$ function is

$$
\begin{aligned}
& \mathrm{F}_{2}\left[\begin{array}{c}
a, b,-m \\
d, e
\end{array}\right] \\
& \quad=(-1)^{m} \Gamma\left[\begin{array}{c}
1-d+b, d \\
d+m, 1+b-m-d
\end{array}\right]{ }_{3} \mathrm{~F}_{2}\left[\begin{array}{c}
e-a, b,-m \\
e, 1+b-m-d
\end{array}\right] .
\end{aligned}
$$

\section{New hypergeometric formulas and reversion}

The purpose of this section is to extend some of the ${ }_{2} \mathrm{~F}_{1}$ formulas of Gessel and Stanton $[2, \S 3]$. In each case, we give the number of the formula in $[2, \S 3]$ followed by two new (old) formulas obtained by reversion (1) or Pfaff-Kummer transformation of the reversion (original for 3.7).

A new summation formula of Pfaff-Kummer based on $[2,3.7]$ :

$$
{ }_{2} \mathrm{~F}_{1}\left[\begin{array}{c|c}
2+\underset{\frac{4}{3}}{2 n,-n} & \frac{8}{9}
\end{array}\right]=(-3)^{n} \frac{\left(\frac{5}{6}\right)_{n}}{\left(\frac{3}{2}\right)_{n}} .
$$

New formulas based on reversed [2, 3.8]:

$$
\begin{gathered}
{ }_{2} \mathrm{~F}_{1}\left[\begin{array}{c|c}
\frac{1}{3}-n,-n & -\frac{1}{8} \\
\frac{1}{3}+n & =\frac{(-3 n+1)_{n} 2^{-n}}{\left(\frac{1}{3},-2 n+\frac{2}{3}\right)_{n}}\left[\left(\frac{1}{6}\right)_{n}+\frac{1}{2}\left(\frac{1}{2}\right)_{n}\right], \\
{ }_{2} \mathrm{~F}_{1}\left[\begin{array}{c}
2 n,-n \\
\frac{1}{3}+n
\end{array} \mid \frac{1}{9}\right.
\end{array}\right]=\frac{(-3 n+1)_{n} 4^{n}}{\left(\frac{1}{3},-2 n+\frac{2}{3}\right)_{n} 9^{n}}\left[\left(\frac{1}{6}\right)_{n}+\frac{1}{2}\left(\frac{1}{2}\right)_{n}\right] .
\end{gathered}
$$

New formulas based on reversed $[2,3.9]$ :

$$
\begin{aligned}
& { }_{2} \mathrm{~F}_{1}\left[\begin{array}{c|c}
\frac{2}{3}-n,-n \\
-\frac{1}{3}+n
\end{array} \mid-\frac{1}{8}\right]=\frac{(-3 n+2)_{n}}{\left.\left(-\frac{1}{3},-2 n+\frac{4}{3}\right)\right)_{n} 2^{n}}\left[\left(-\frac{1}{6}\right)_{n}+\frac{1}{2}\left(-\frac{1}{2}\right)_{n}\right], \\
& { }_{2} \mathrm{~F}_{1}\left[\begin{array}{c}
-1+2 n,-n \\
-\frac{1}{3}+n
\end{array} \mid \frac{1}{9}\right]=\frac{(-3 n+2)_{n} 4^{n}}{\left(-\frac{1}{3}, \frac{4}{3}-2 n\right)_{n} 9^{n}}\left[\left(-\frac{1}{6}\right)_{n}+\frac{1}{2}\left(-\frac{1}{2}\right)_{n}\right] .
\end{aligned}
$$

New formulas based on reversed [2, 3.10]:

$$
\begin{aligned}
& { }_{2} \mathrm{~F}_{1}\left[\begin{array}{c|c}
\frac{4}{3}-n,-n \\
-\frac{5}{3}+n & -\frac{1}{8}
\end{array}\right] \\
& =\frac{(-3 n+4)_{n}}{\left(-2 n+\frac{8}{3},-\frac{2}{3}\right)_{n} 2^{n}}\left[\left(-\frac{5}{6}\right)_{n}+\left(\frac{n}{3}-\frac{3}{4}\left(-\frac{1}{2}\right)_{n-1}\right], n \geq 2,\right. \\
& { }_{2} \mathrm{~F}_{1}\left[\begin{array}{c}
-3+2 n,-n \\
-\frac{5}{3}+n
\end{array} \mid \frac{1}{9}\right] \\
& =\frac{(-3 n+4)_{n} 4^{n}}{\left(-2 n+\frac{8}{3},-\frac{2}{3}\right)_{n} 9^{n}}\left[\left(-\frac{5}{6}\right)_{n}+\left(\frac{n}{3}-\frac{3}{4}\right)\left(-\frac{1}{2}\right)_{n-1}\right], n \geq 2 .
\end{aligned}
$$

Heymann [3], based on [2, 3.12]:

$$
{ }_{2} \mathrm{~F}_{1}\left[\begin{array}{c|c}
\frac{2}{3}+n,-\frac{1}{2} n+\frac{1}{6} & \frac{8}{9}
\end{array}\right]=2 \cdot 3^{\frac{1}{2} n-\frac{1}{6}} \cos \left(n \frac{1}{2} \pi+\frac{1}{6} \pi\right), n \geq 2 .
$$


A new formula based on $[2,3.13]$ :

$$
\begin{aligned}
& { }_{2} \mathrm{~F}_{1}\left[\begin{array}{c|c}
\frac{1}{3}-\frac{1}{2} n,-n & -8 \\
\frac{1}{3} & -8
\end{array}\right]=\frac{\left(-\frac{1}{2} n+\frac{1}{3}\right)_{n}}{\left(\frac{1}{3}\right)_{n}} 8^{n}{ }_{2} \mathrm{~F}_{1}\left[\begin{array}{c|c}
\frac{2}{3}-n,-n & -\frac{1}{8} \\
\frac{2}{3}-\frac{1}{2} n & -1
\end{array}\right] \\
& =\frac{\left(-\frac{1}{2} n+\frac{1}{3}\right)_{n}}{\left(\frac{1}{3}\right)_{n}} 9^{n}{ }_{2} \mathrm{~F}_{1}\left[\begin{array}{c|c}
\frac{1}{2} n,-n & \frac{1}{2} \\
\frac{2}{3}-\frac{1}{2} n & 9
\end{array}\right] .
\end{aligned}
$$

A formula based on reversed [2, 3.14]:

$$
{ }_{2} \mathrm{~F}_{1}\left[\begin{array}{c|c}
-\frac{1}{3}-2 N,-2 N & -\frac{1}{8}
\end{array}\right]=\frac{3^{3 N}(-1)^{N}\left(\frac{4}{3}\right)_{2 N}}{\frac{7}{6}-N} .
$$

A new summation formula of Pfaff-Kummer, which is based on [2, 3.14]:

$$
{ }_{2} \mathrm{~F}_{1}\left[\begin{array}{c|c}
\frac{3}{2}+N,-2 N & \frac{1}{9} \\
\frac{7}{6}-N & 9
\end{array}\right]=\frac{3^{-N}(-1)^{N}\left(\frac{4}{3}\right)_{2 N}}{(2 N+1)\left(-\frac{1}{6}-N\right)_{2 N}} .
$$

Formulas based on reversed [2, 3.15]:

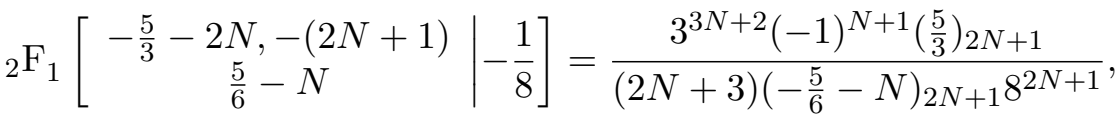

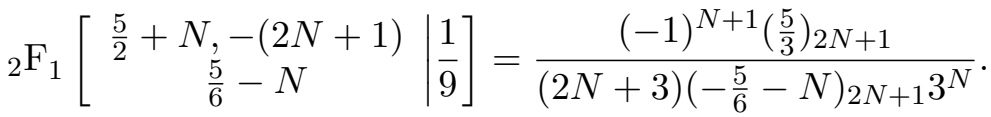

A formula based on reversed [2, 3.16]:

$$
{ }_{2} \mathrm{~F}_{1}\left[\begin{array}{c|c}
-\frac{7}{3}-2 N,-(2 N+1) & -\frac{1}{8} \\
\frac{7}{6}-N
\end{array}\right]=5 \frac{3^{3 N+2}(-1)^{N+1}\left(\frac{7}{3}\right)_{2 N+1}}{(2 N+3)(2 N+5)\left(-\frac{7}{6}-N\right)_{2 N+1} 8^{2 N+1}} .
$$

A new summation formula of Pfaff-Kummer based on [2, 3.16]:

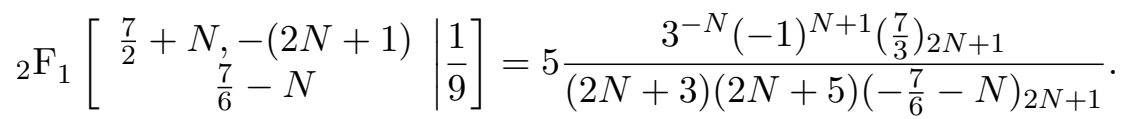

A formula based on reversed $[2,3.17]$ :

$$
\begin{aligned}
9^{n} & { }_{3} \mathrm{~F}_{2}\left[\begin{array}{c}
-\frac{2}{3} n, \frac{1}{3}-\frac{2}{3} n, \frac{2}{3}-\frac{2}{3} n \\
-n+\frac{1}{2}, 1-a-n
\end{array}\right] \\
\quad= & \frac{(2 a)_{n}}{(1-a-n)_{n}}(-4)^{n}{ }_{2} \mathrm{~F}_{1}\left[\begin{array}{c}
a,-n \\
1-2 a-n
\end{array}\right.
\end{aligned}
$$

The ${ }_{3} \mathrm{~F}_{2}$ series is balanced for $a=-\frac{1}{2}$.

Example 1. Put $a=1-\frac{1}{3} n$ in (15) to get

$$
\begin{aligned}
& { }_{2} \mathrm{~F}_{1}\left[\begin{array}{c|c}
1-\frac{1}{3} n,-n & \frac{1}{4} \\
-1-\frac{1}{3} n &
\end{array}\right] \\
& \quad=\left(-\frac{9}{4}\right)^{n} \Gamma\left[\begin{array}{c}
\frac{1}{3} n, 2-\frac{1}{3} n, \frac{1}{2}-n,-\frac{1}{2}+\frac{1}{3} n \\
-\frac{2}{3} n, 2+\frac{1}{3} n, \frac{1}{6}+\frac{1}{3} n,-\frac{1}{6}+\frac{1}{3} n
\end{array}\right], 3 \nmid n .
\end{aligned}
$$


Example 2. Put $a=\frac{2}{3}-\frac{1}{3} n$ in (15) to get

$$
\begin{aligned}
& { }_{2} \mathrm{~F}_{1}\left[\begin{array}{c|c}
\frac{2}{3}-\frac{1}{3} n,-n & \frac{1}{3} \\
-\frac{1}{3}-\frac{1}{3} n & \frac{1}{4}
\end{array}\right] \\
& \quad=\left(-\frac{9}{4}\right)^{n} \Gamma\left[\begin{array}{c}
\frac{1}{3}+\frac{1}{3} n, \frac{4}{3}-\frac{2}{3} n, \frac{1}{2}-n,-\frac{1}{6}+\frac{1}{3} n \\
\frac{1}{3}-\frac{2}{3} n, \frac{4}{3}+\frac{1}{3} n, \frac{1}{2}-\frac{1}{3} n,-\frac{1}{6}-\frac{1}{3} n
\end{array}\right], 3 \nmid n .
\end{aligned}
$$

A formula based on $[2,3.18]$ :

$$
\begin{aligned}
3^{n} & { }_{3} \mathrm{~F}_{2}\left[\begin{array}{c}
-\frac{1}{3} n, \frac{1}{3}-\frac{1}{3} n, \frac{2}{3}-\frac{1}{3} n \\
a+\frac{1}{2}, 1-2 a-n
\end{array}\right] \\
\quad= & \frac{(a)_{n}}{(1-2 a-n)_{n}}(-4)^{n}{ }_{2} \mathrm{~F}_{1}\left[\begin{array}{c|c}
2 a,-n \\
1-a-n & \frac{1}{4}
\end{array}\right] \\
\quad= & \frac{(a)_{n}}{(1-2 a-n)_{n}}(-3)^{n}{ }_{2} \mathrm{~F}_{1}\left[\begin{array}{c|c}
1-3 a-n,-n & 1 \\
1-a-n & -\frac{3}{3}
\end{array}\right] .
\end{aligned}
$$

4. Extensions of $[2, \S 6]$

In this section we show some corollaries of conjectures made in $[2, \S 6]$. Conjecture based on $[2,6.5]$ :

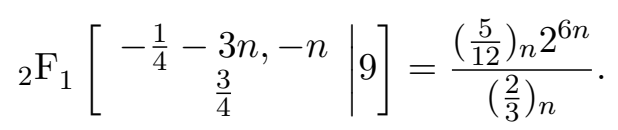

Proof. We begin with reversion

$$
\begin{aligned}
& \frac{\left(\frac{5}{4}\right)_{2 n}}{\left(\frac{2}{3}, \frac{13}{12}\right)_{n}}\left(\frac{2^{6}}{3^{5}}\right)^{n}=\left(-\frac{1}{9}\right)^{n} \frac{\left(\frac{1}{4}-n\right)_{n}}{\left(2 n+\frac{5}{4}\right)_{n}}{ }_{2} \mathrm{~F}_{1}\left[\begin{array}{c|c}
-\frac{1}{4}-3 n,-n \\
\frac{3}{4}
\end{array} \mid 9\right] \\
& =\frac{\left(\frac{3}{4}\right)_{n}\left(\frac{5}{4}\right)_{2 n}}{9^{n}\left(\frac{5}{4}\right)_{3 n}}{ }_{2} \mathrm{~F}_{1}\left[\begin{array}{c|c}
-\frac{1}{4}-3 n,-n & 9 \\
\frac{3}{4} &
\end{array}\right] \\
& =\frac{\left(\frac{3}{4}\right)_{n}\left(\frac{5}{4}\right)_{2 n}}{3^{2 n} 3^{3 n}\left(\frac{5}{12}, \frac{9}{12}, \frac{13}{12}\right)_{n}}{ }_{2} \mathrm{~F}_{1}\left[\begin{array}{c|c}
-\frac{1}{4}-3 n,-n & 9 \\
\frac{3}{4} & 9
\end{array}\right] \\
& =\frac{\left(\frac{5}{4}\right)_{2 n}}{3^{5 n}\left(\frac{5}{12}, \frac{13}{12}\right)_{n}}{ }_{2} \mathrm{~F}_{1}\left[\begin{array}{c|c}
-\frac{1}{4}-3 n,-n \\
\frac{3}{4}
\end{array} \mid 9\right] .
\end{aligned}
$$

Finally, solve for ${ }_{2} \mathrm{~F}_{1}$.

We continue with a couple of formulas valid for complex $a$ :

$$
\begin{gathered}
{ }_{2} \mathrm{~F}_{1}\left[\begin{array}{c|c}
1+3 a,-a & -\frac{1}{8} \\
\frac{5}{4}+2 a & =\frac{\left(\frac{5}{4}\right)_{2 a} 2^{3 a}}{\left(\frac{2}{3}, \frac{13}{12}\right)_{a} 3^{3 a}}, \\
{ }_{2} \mathrm{~F}_{1}\left[\begin{array}{c|c}
\frac{5}{4}+3 a, 3 a+1 \\
\frac{5}{4}+2 a
\end{array} \mid \frac{1}{9}\right.
\end{array}\right]=\frac{\left(\frac{5}{4}\right)_{2 n} 3^{3 a+2}}{\left(\frac{2}{3}, \frac{13}{12}\right)_{a} 8^{2 a+1}} .
\end{gathered}
$$


Proof. By Pfaff-Kummer transformation we get

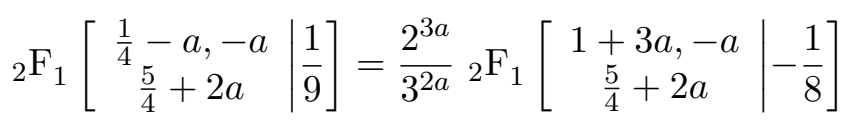

which gives (16).

By Euler transformation we obtain

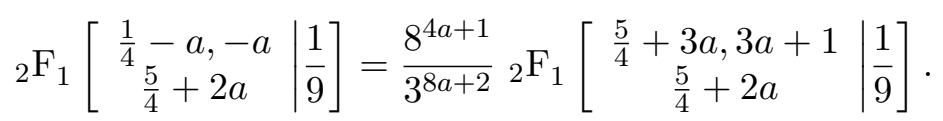

This proves (17).

Conjectures based on $[2,6.6]$ :

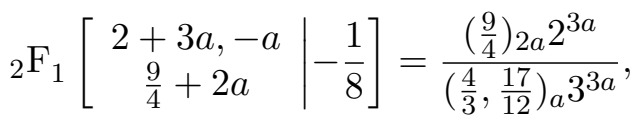

$$
\begin{aligned}
& { }_{2} \mathrm{~F}_{1}\left[\begin{array}{c|c}
\frac{9}{4}+3 a,-a+\frac{1}{4} & -\frac{1}{8}
\end{array}\right]=\frac{\left(\frac{9}{4}\right)_{2 a} 2^{3 a+\frac{3}{4}}}{\left(\frac{4}{3}, \frac{17}{12}\right)_{a} 3^{3 a+\frac{1}{2}}},
\end{aligned}
$$

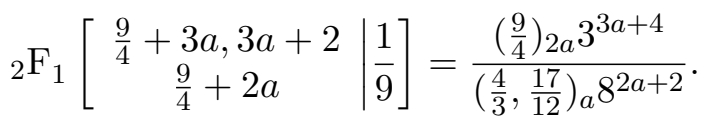

Proof. Use Pfaff-Kummer transformation twice, and finally, use Euler transformation.

\section{Conclusion}

Our method of proof is not widely spread. The reason that Pfaff-Kummer transformation and Euler transformation work for function arguments greater than 1 is that the series are terminating. It was not possible to find any of these summation formulas in the standard reference work [6]. This technique could also be used for terminating $q$-hypergeometric series.

\section{References}

[1] W. N. Bailey, Generalized Hypergeometric Series, Cambridge Tracts in Mathematics and Mathematical Physics 32, Stechert-Hafner, New York, 1964.

[2] I. Gessel and D. Stanton, Strange evaluations of hypergeometric series, SIAM J. Math. Anal. 13 (1982), 295-308.

[3] W. Heymann, Über hypergeometrische Functionen, deren letztes Element speciell ist, nebst einer Anwendung auf Algebra, Chemnitz, 1898.

[4] C. Lal, An integral involving Kampé de Fériet function, Math. Notae 24 (1974/75), $19-22$.

[5] M. A. Melvin and N. V. V. J. Swamy, Evaluation of certain physically interesting integrals and hypergeometric sums, J. Math. and Phys. 36 (1957), 157-163.

[6] A. P. Prudnikov, Yu. A. Brychkov, and O. Marichev, Integrals and Series. Vol. 3. More Special Functions, Translated from the Russian by G. G. Gould, Gordon and Breach Science Publishers, New York, 1990. 
[7] L. J. Slater, Generalized Hypergeometric Functions, Cambridge University Press, Cambridge, 1966.

[8] H. M. Srivastava and P. W. Karlsson, Multiple Gaussian Hypergeometric Series, Halsted Press (Ellis Horwood Limited, Chichester), John Wiley and Sons, New York, Chichester, Brisbane, and Toronto, 1985.

[9] V. F. Tarasov and S. M. Čanyšev, The deduction of Balmer's formula from a generalized hypergeometric differential equation, Izv. Akad. Nauk Kazah. SSR Ser. Fiz.-Mat., no. 6 (1970), 59-62. (Russian)

Department of Mathematics, Uppsala University, P.O. Box 480, SE-751 06 UPPSALA, SWEDEN

E-mail address: thomas@math.uu.se 